\title{
Is axenicity crucial to cryopreserve microalgae? is
}

\author{
Raquel Amaral $^{\mathrm{a}, *}$, Jorge C. Pereira ${ }^{\mathrm{b}}$, Alberto A.C.C. Pais ${ }^{\mathrm{b}}$, Lília M.A. Santos ${ }^{\mathrm{a}}$ \\ a Algoteca do Departamento de Ciências da Vida (Botânico), Universidade de Coimbra, 3001-455 Coimbra, Portugal \\ ${ }^{\mathrm{b}}$ Departamento de Química, Faculdade de Ciências e Tecnologia, Universidade de Coimbra, 3004-535 Coimbra, Portugal
}

\section{A R T I C L E I N F O}

\section{Article history:}

Received 22 February 2013

Accepted 11 September 2013

Available online 20 September 2013

\section{Keywords:}

Axenicity

Cryopreservation

Microalgae

Partner organisms

Pop

\begin{abstract}
A B S T R A C T
Large culture collections of microalgae and cyanobacteria such as the Coimbra Collection of Algae (ACOI) hold unialgal cultures consisting of a population of cells/colonies of a certain species. These cultures are usually non-axenic, as other organisms such as bacteria and microfungi are also present in culture due to co-isolation. Attention has been recently given to partner organisms since studies indicate that some bacteria are important for nutrient uptake of the algal cells, acting as simbionts. Despite this benign effect in the actively growing cultures, when cryopreservation is applied for inactive-stage storage, these organisms may recover faster than the algae, thus affecting their recovery and the viability assessments. In this study, a set of mucilaginous ACOI microalgae were selected, cell features known for their relevance in cryopreservation success were recorded and simple two-step cryopreservation tests were applied. Thawed samples were transferred to fresh culture medium for recovery. Viability was assessed and partner organism proliferation (pop) was recorded. Results were analyzed by $t$-tests. Statistical models allowed us to support the known tendency for small, unicellular algae with no outer structures to be successfully cryopreserved and the negative effect of vacuoles in the cell prior to cryopreservation. On average cryopreservation with $\mathrm{MeOH}$ or $\mathrm{Me}_{2} \mathrm{SO}$ led to the recovery of nearly half the cells. It was found that the cryoprotection step with $\mathrm{MeOH}$ is when pop is triggered and that the use of $\mathrm{Me}_{2} \mathrm{SO}$ can prevent this effect. Progress on understanding the cultured consortia will assist the improvement of cryopreservation and research using microalgal cultures.
\end{abstract}

(c) 2013 Elsevier Inc. All rights reserved.

\section{Introduction}

Many large culture collections of microalgae and cyanobacteria keep their holdings in a non-axenic state. Non-axenicity implies the presence in culture of organisms other than algae, even in such low numbers that they are not visible to the naked eye. These partner organisms consist mainly of bacteria and microfungi, whose proliferation is disadvantaged by the use of culture media with algal-specific formulas [29]. The isolation and cultivation of microalgae became a broad activity in 1960-70 with the work of researchers, who established culture collections such as ACOI [29]. Attempts to remove the co-isolates were developed by mechanical methods and also by the use of antibiotics, but the latter was criticized. The reasons were the difficulty of maintaining viable cultures cleaned by antibiotics [11] and the cell damage inflicted by the antibiotic action, together with the observation that sometimes axenicity was not fully achieved [18]. Important progress in understanding the dynamics of nutrient uptake by

\footnotetext{
Statement of funding: The funding bodies involved in the research published in the submitted manuscript are public science and education entities, so there is nothing to declare regarding any incompatible archiving policy.

* Corresponding author. Fax: +351239855211.

E-mail address: raquel.amaral@bot.uc.pt (R. Amaral).
}

the microalgae growing in batch culture and chemostat was achieved by M.R. Droop's studies on cobalamin (vitamin $\mathrm{B}_{12}$ ) uptake by a symbiotic interaction of the algal cells with co-cultivated bacteria. His "cell-quota model" of phytoplankton growth relates the microalgal growth rate to the internal nutrient content of the cell, in this case, the nutrient is vitamin $B_{12}$ ([19] and references therein). This prompted the suggestion that the reason for the failure in cultivating pelagic diatoms without the associated bacteria was a "possible obligatory relationship" [12]. The most common symbiotic interaction shown to occur for $\mathrm{B}_{12}$ uptake was observed in at least half of 326 surveyed microalgae from different groups [7]. Nevertheless, other nutrients are also made available by simbiont partners. The study of dinoflagelates in oceanographic dynamics research revealed a singular ability of their symbiotic bacteria to enhance iron uptake [3]. The community composition of co-isolates is starting to be revealed by molecular studies that reinforce the potential of non-axenic cultures as material for studies on algal-bacteria associations [32] that may be useful for the fermented food industries and waste water treatment [34]. The symbiotic interaction between microorganisms and photosynthetic organisms is still unclear and it is not restricted to microalgae. It has also been observed in macroalgae [26] and in land plants, sometimes with a chain of mutualists involved such as the triplet virus-fungus-plant associations in geothermal soils [21]. 
Years of observations of algae belonging to a broad range of taxa in the ACOI collection show that partner organisms such as bacteria and fungi are either distributed in the culture medium or embedded in the mucilage produced by the algae. This last case provides a robust scenario for studying the effects of cryopreservation stresses in non-axenic cultures, because partner organisms live very closely to the algal cells, it is also probable that a symbiotic relationship is present. The sterilization of all glassware and the handling of the cultures at all times in the flow chamber prevents the contamination of the culture with organisms not belonging to the isolated microbial consortia. Regarding the culture media, quite often the formulas include the addition of vitamins. The preparation recipes for vitamin solutions are either provided by culture collections in their websites or in published methods and lists [30] and usually there is an indication that stock vitamins should be prepared separately and filter sterilized prior to use. Paradoxically, in most cases, the general recipe of the culture media includes the vitamin stock as part of the stock solutions to be added to the final mixture, later autoclaved at $120^{\circ} \mathrm{C}$. This probably leads to total or partial disintegration of the vitamins, since most of them are heat-sensitive. This is not fully the case of vitamin $B_{12}$, which is generally considered as heat stable. Nevertheless, like all water-soluble vitamins, some disintegration may occur on heating if they are dissolved in large volumes of water [20]. When included in $1 \mathrm{~L}$ of culture medium to be autoclaved, unpredictable loss may occur and cobalamin-auxotrophs developing in such culture medium, may suffer from vitamin deficiency. This aspect reinforces the importance of partners with their role as cobalamin providers.

The decision of keeping the co-isolates is based on these arguments and also on the concern that the use of detergents, antibiotics or other chemicals for their removal may cause selection of resistant microalgal populations and genetic erosion. Despite the reasons for keeping non-axenic cultures, the presence of bacteria and fungi may become problematic when cryopreservation stress is imposed on the cultures. Algal cells may require more time to recover than their partner organisms and this may result in a higher risk of their proliferation after thawing [9]. One month after recovering from cryopreservation procedures, some thawed samples show a great level of opacity denoting pop, which spreads throughout the culture [1]. One of the causes for this exacerbation is the use of sugar-based cryoprotectants, a preferential carbon source for heterotrophic partners. An extra source of nutrients may also be the release of cellular content as a result of cell lysis caused by cryoinjury [5].

The 'cell vigor' of the microalgal culture prior to cryopreservation is considered as a key-factor for its success [6]. It is generally accepted that cryopreserved cultures in the actively growing phase show better viability after thawing than those that were in the stationary phase, or growing under stress conditions before being cryopreserved [9]. Also, previous reports indicate that strains displaying specific cell characteristics are usually highly viable after thawing. These features include microalgae which are unicellular, small, spherical, with no spines and without vacuoles at the time of cryopreservation (e.g. [27,1]).

Assessments of the dynamics and effects of pop in cryopreserved cultures are complex since they must take into consideration the importance of the cell characteristics in relation to cryopreservation stress. It is desirable that these aspects be studied, together with the occurrence of pop in a comprehensive approach to determine when and how pop impacts take place.

The two-step cryopreservation method developed by Morris [23] has been broadly applied to cryopreserve microalgal cultures except for large, sensitive or fragile species. In the case here, the microalgae are usually cryopreserved by encapsulation/dehydration, a method developed by Hirata et al. for higher plant material [16], which now is quite often used to overcome recalcitrance in microalgae, although it promotes high proliferation of partner organisms due to the use of sugar-based cryoprotectants [5].

For collections with large holdings waiting to be cryopreserved, including species with no previous cryopreservation history published in the literature, it is useful to develop standard approaches. This is done at ACOI, where sets of microalgal cultures are cryopreserved using a step-by-step approach designed to draw the best possible protocol for each strain (Fig. 1). After the sequence of tests is applied but no suitable protocol for permanent storage is found for a strain, changes in the culture conditions are considered. This is also done if good viability is achieved but the culture shows too lower cell density to consider permanent storage. The cell density of the culture may be enhanced for example by raising the $\mathrm{pH}$

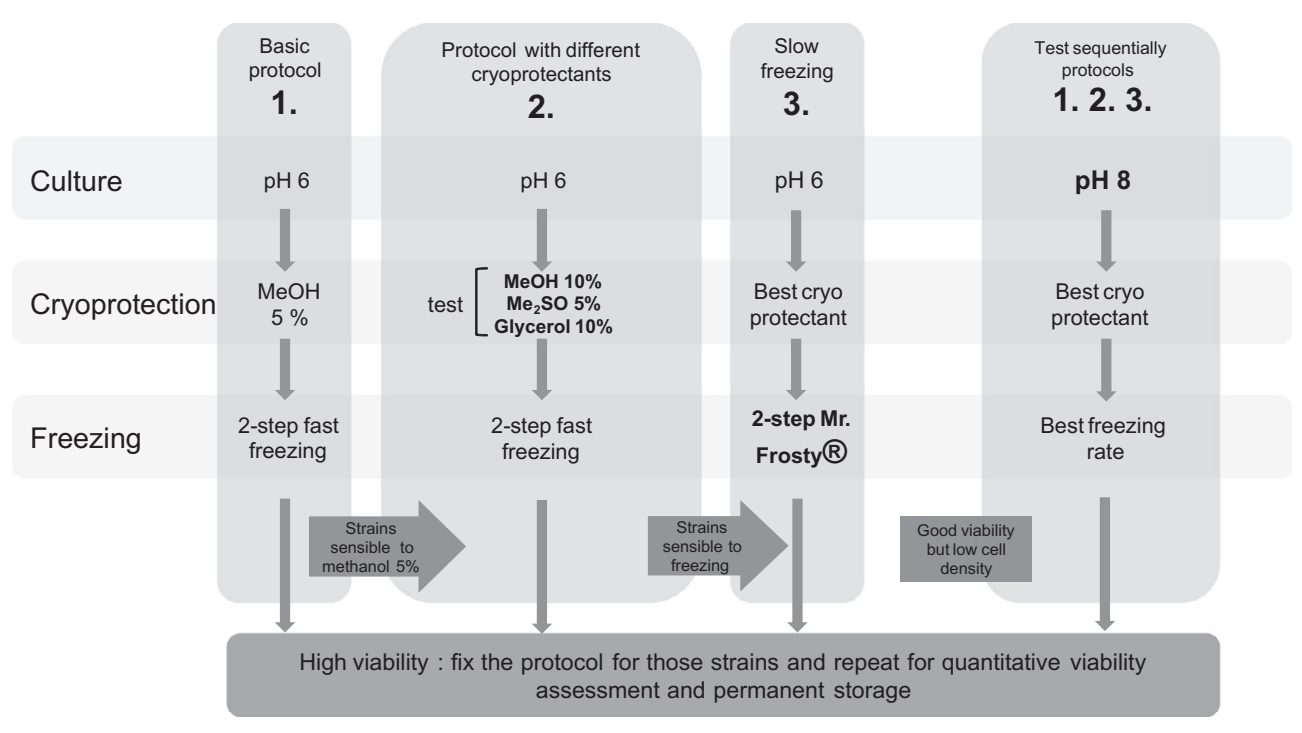

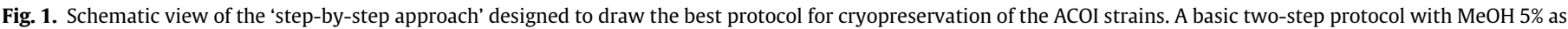

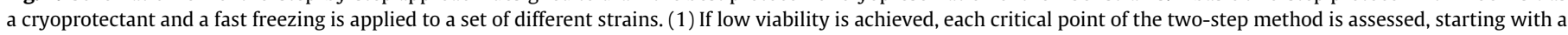

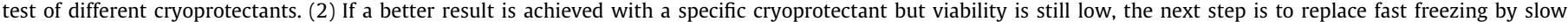

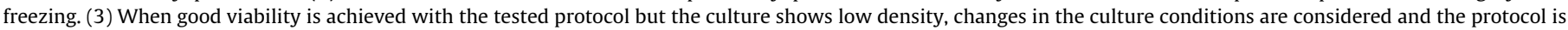
applied to a denser culture. When high viability $(>50 \%)$ is achieved with one of the tested protocols, permanent storage is possible. 
value of the culture medium. This chain of tests creates tailor-made protocols for sets of different microalgae to be cryopreserved.

In this study, mucilaginous microalgae were cryopreserved and several questions were addressed, due to the consistent observation of covert organism proliferation after thawing: (1) if pop affects the recovery of algal cells; (2) if there is a specific step of the protocol promoting pop; and (3) if axenicity of cultures is crucial when cryopreserving algal cultures.

\section{Materials and methods}

\section{Selection of strains and culture conditions}

Nine unialgal, non-axenic ACOI strains, known to produce great amounts of mucilage (Table 1) were selected from a catalogue of ACOI mucilaginous microalgae. Strains were observed prior to cryopreservation and classified as: 'unicellular'/'other', 'small'/ 'large', 'no vacuoles observed'/'with vacuoles'. In the case of colonial algae disintegrating at the time of cryopreservation, they were classified as unicellular.

The strains were cultivated for $34 \pm 3$ days in small glass tubes with $20 \mathrm{ml}$ desmidiacean medium [30], with pH adjusted to 6, under a 12:12 h light:dark regime, provided by cool white fluorescent lamps with a photon flux density of $60 \mu \mathrm{mol} \mathrm{m}{ }^{-2} \mathrm{~s}^{-1}$ and $18{ }^{\circ} \mathrm{C}$ air temperature.

Materials were always sterile and all work was performed in a flow chamber according to general microbiological practice for the aseptic manipulation of cultured organisms, in order to ensure that no organisms from the outside environment contaminated the culture.

\section{Cryopreservation}

The cryoprotectant solution was previously prepared in culture medium at twice the required final concentration of $5 \%(\mathrm{v} / \mathrm{v})$. The cultures were cryopreserved following the method illustrated in Fig. 2. A volume of $0.5 \mathrm{ml} 10 \%(\mathrm{v} / \mathrm{v}) \mathrm{MeOH}$ was added to $0.5 \mathrm{ml}$ of a dense microalgal culture in a cryovial. The mixture was incubated for $15 \mathrm{~min}$ on ice and low light and periodically mixed during incubation. Samples were then frozen using a two-step procedure. The first step consisted of rapid freezing to $-80^{\circ} \mathrm{C}$ and holding for 45-60 min in the freezer; the second step consisted of plunging the samples into liquid nitrogen (LN). After $30 \mathrm{~min}$ the cryovials were thawed in a preheated water bath at $40^{\circ} \mathrm{C}$, for 1-2 min, until no ice crystals were observed and then rapidly transferred to the flow cabinet. The content of each thawed cryovial was then inoculated in a tube with $20 \mathrm{ml}$ of liquid medium for recovery, labeled as ' $S$ ' (sample). Two types of control tubes

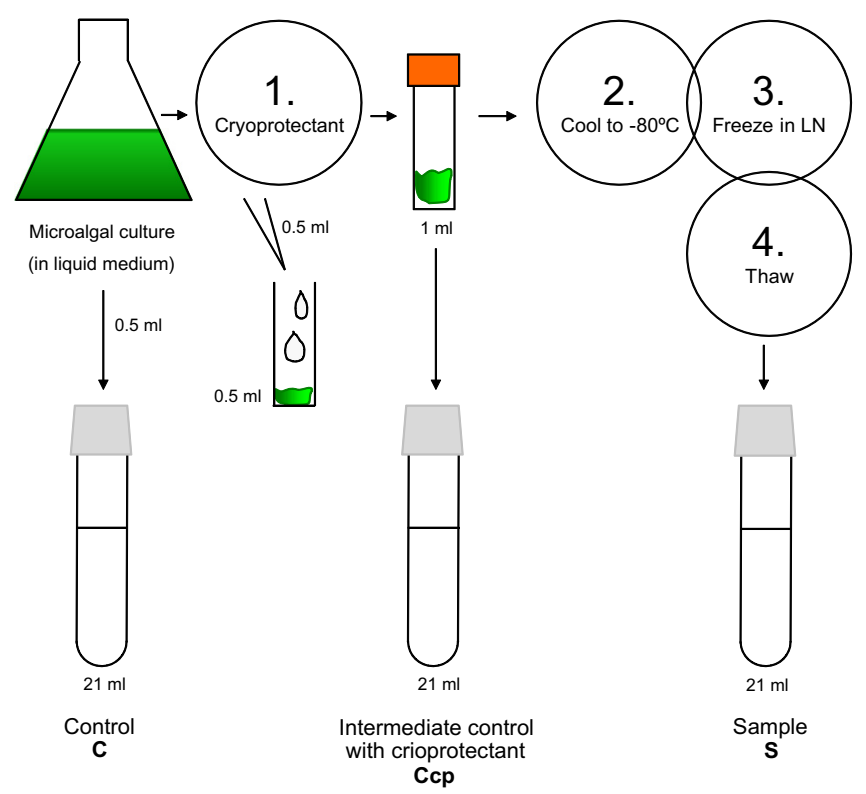

Fig. 2. Diagram illustrating how control tubes were prepared. C - control tube, untreated culture; $\mathrm{Ccp}$ - intermediate control tube, culture with cryoprotectant; S samples, culture that underwent the complete cryopreservation procedure. 1 addition of cryoprotectant; 2 - rapid cooling step; 3 - plunging into liquid nitrogen (LN); 4 - thawing at $40{ }^{\circ} \mathrm{C}$ and transfer into culture medium.

were established: one without any treatment, consisting of $0.5 \mathrm{ml}$ of the culture in $20.5 \mathrm{ml}$ of culture medium, labeled as ' $\mathrm{C}$ ' (control); another after cryoprotection, before freezing, consisting of $1 \mathrm{ml}$ of the mixture culture-cryoprotectant transferred to a recovery tube with $20 \mathrm{ml}$ culture medium, labeled as 'Ccp' (control after cryoprotectant addition). Samples (S) and controls (C \& Ccp) were kept in the dark overnight to prevent photo-oxidation [13] and then transferred to standard culture conditions to grow for subsequent viability assessments. Pop was evaluated at each treatment level (C, Ccp and $S$ ) in all tubes. This experiment was repeated 3 times. An alternative cryoprotectant, dimethylsulfoxide $\left(\mathrm{Me}_{2} \mathrm{SO}\right)$, was also tested.

\section{Viability and pop assessment}

Samples and controls were incubated in the culture room at standard conditions as previously mentioned. Recovery was evaluated after 20-30 days, based on the color intensity displayed by the samples in comparison with controls, similar color of both samples

\section{Table 1}

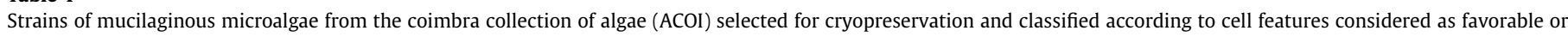
unfavorable to cryopreservation.

\begin{tabular}{|c|c|c|c|}
\hline \multirow[t]{2}{*}{ ACOI number } & \multirow[t]{2}{*}{ Taxa } & \multicolumn{2}{|l|}{ Cell features } \\
\hline & & Favorable to cryopreservation & Unfavorable to cryopreservation \\
\hline ACOI 312 & Dictyosphaerium pulchellum Wood & Small & Colonial, with vacuoles \\
\hline ACOI 480 & Tetrallantos lagerheimii Teiling & Small, no vacuoles & Colonial \\
\hline ACOI 1831 & Quadrigula sabulosa Hindák & Small, no vacuoles & Colonial \\
\hline ACOI 1847 & Cryptomonas pyrenoidifera Geitler & Unicellular & Large, with vacuoles \\
\hline ACOI 2200 & Radiococcus planktonicus Lund & Unicellular ${ }^{\mathrm{a}}$, small & With vacuoles \\
\hline ACOI 2331 & Aphanothece sp. Nageli & Small, no vacuoles & Colonial \\
\hline ACOI 2507 & Kirchneriella sp. Schmidle & Unicellular ${ }^{\mathrm{a}}$, small, no vacuoles & - \\
\hline ACOI 2771 & Cosmarium sp. Corda & Unicellular, no vacuoles & Large \\
\hline ACOI 1767 & Porphyridium sordidum Geitler & Unicellular, small, no vacuoles & - \\
\hline
\end{tabular}

Strains were collected from diverse habitats in Portugal except ACOI 1847, collected at São Tomé e Príncipe (visit http://acoi.ci.uc.pt for more information on strains).

${ }^{a}$ It is colonial or it aggregates but disintegrates to isolated cells during the treatments so for the purpose of the study it was treated as unicellular. 
and controls indicated the recovery of frozen cells. A score strategy was used in a semi-quantitative approach to assess viability, evaluating the ability of cells to actively divide in liquid medium (activity) after cryopreservation, to recover to a normally growing culture. The color evaluation system used was calibrated by classifying the control tube ' $C$ ' according to the density of the motherculture (see Fig. 2) and its score was considered as the maximal growth for each strain in the experiment. The intermediate control, 'Ccp' and sample ' $S$ ', were evaluated by direct comparison with the corresponding control tube ' $\mathrm{C}$ ' and classified according to the color intensity (green) displayed on the bottom of the tube. Total cell activity loss was scored as ' 0 ' while activity similar to the control was scored as '5'. Similarly, culture opacity (white) caused by pop on the culture medium throughout the tube, was evaluated in all tubes (corresponding to 'popC', 'popCcp' and 'popS'), ranging from ' 0 ' (no pop observed) to ' 3 ' (highest level of proliferation). Triplicates were used at every experimental level to provide statistical significance.

To obtain the cell activity and pop relative response for all tested strains, the data collected for each strain were treated as a group and the \% activity value was obtained by comparison with the average of the ' $\mathrm{C}$ ' obtained for all strains. The resulting percentage values show the \% cell activity and pop in the tested strains, which is extrapolated as representative of these responses in mucilaginous strains.

\section{Statistical analysis}

Throughout the statistical study the quantified variables were defined as factors and responses. The factors were the experimentally controlled conditions that had a measured effect, namely, the addition and type of cryoprotectant $\left(\mathrm{MeOH}\right.$ and $\mathrm{Me}_{2} \mathrm{SO}$ ) and the steps of the cryopreservation protocol (cryoprotectant addition and freezing/thawing). The parameters considered as responses were the measured performances, namely algal activity (C, Ccp, $\mathrm{S}$ ) and pop (popC, popCcp, popS) following the previously described score strategy.

For the multivariate modeling the evaluated cell features were also considered as factors after being coded as: ' 1 ' designated for unicellular algae (uni.) and ' 0 ' for colonial; ' 1 ' for small (small) and ' 0 ' for large cells; ' 1 ' for presence (vac.) and ' 0 ' for absence/ undetected vacuoles. Since algal activity and pop data were recorded at different scale ranks, these data were also previously scaled into a 0-1 range, to directly establish the relevance of each feature according to the magnitude of the estimated sensitivity coefficient.

Firstly, to evaluate the differences between the cryoprotectants ( $\mathrm{MeOH}$ and $\mathrm{Me}_{2} \mathrm{SO}$ ) along the cryopreservation process, paired $t$-tests were conducted to check result equality $\left(H_{0}\right)$. Null hypothesis acceptance $(p \geqslant 0.05)$ and rejection $(p<0.01)$ criteria were verified on the basis of the respective probability calculation, $p$-value. Dubious cases $(0.01 \leqslant p<0.05)$ were also considered as $H_{0}$ rejection.

Secondly, to access the cause-effect relations of the factors on the responses, a multivariate modeling strategy was implemented for direct factor analysis using a multilinear least squares approach:

$R=f(x)=b_{0}+\sum_{i=1}^{m-1} b_{i} x_{i}$

in which $R$ is the predicted response, $x$ is the vector of factor variables $x_{\mathrm{i}}, b_{0}$ is the independent term and the remaining parameter, $b_{\mathrm{i}}$, corresponds to $m-1$ factor sensitivity coefficients.

The purpose of modeling was to statistically evaluate what was affecting algal activity (Ccp, S) and the pop observations (popCcp, popS) after the cryoprotection step, regarded as responses in Eq. (1), when $\mathrm{MeOH}$ or $\mathrm{Me}_{2} \mathrm{SO}$ were used. All tested models were based on this equation, for which the cellular features (small, vac. and uni.) were always used as independent variables. The response dependencies related to Ccp and S were incorporated in the model development. Since cryopreservation is a sequential process (represented by C, Ccp and S), the independent variables for algal activity and pop that are associated with Ccp correspond to the cryoprotection step and the step before it (Fig. 3a). In the same way, the independent variables associated with $S$ correspond to the freezing/thawing step and those before it (Fig. 3b).

Before simplification, each response model was assessed with the functional dependence, to describe activity and pop for each cryoprotectant individually, indicated as follows:

Ccp $=f($ small, vac, uni, C, popC, popCcp $)$

popCcp $=f($ small, vac, uni, C, Ccp, popC $)$

$\mathrm{S}=f($ small, vac, uni, C, Ccp, popC, popCcp, popS)

popS $=f($ small, vac, uni, C, Ccp, S, popC, popCcp)

A global model was developed to integrate all experimental information from both cryoprotectants, where cryoprotectant information was added as an extra independent variable in which its presence was coded as ' 1 ' (influence due to $\mathrm{MeOH}$ ) and the absence as ' 0 '. This variable assignment is in agreement with statistical evidences related with the influence of $\mathrm{MeOH}$ promoting pop.

After the response assessment, an ordinary least squares approach [28] was used to estimate the model parameters and respective uncertainty. Model simplification and parameter estimation were iteratively performed starting with a full multivariate

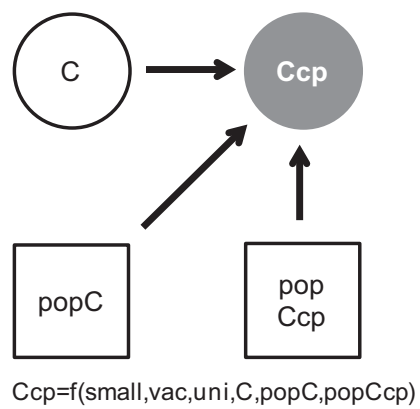

a

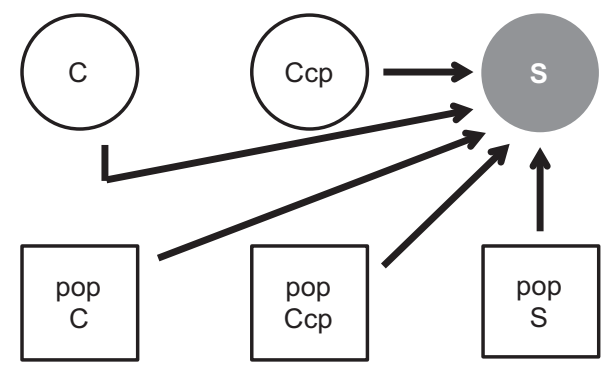

S=f(small,vac,uni,C,Ccp,popC,popCcp,popS)

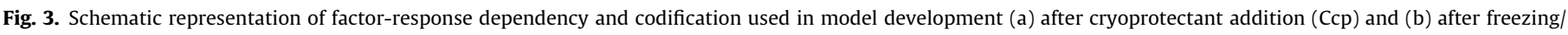
thawing (S). 


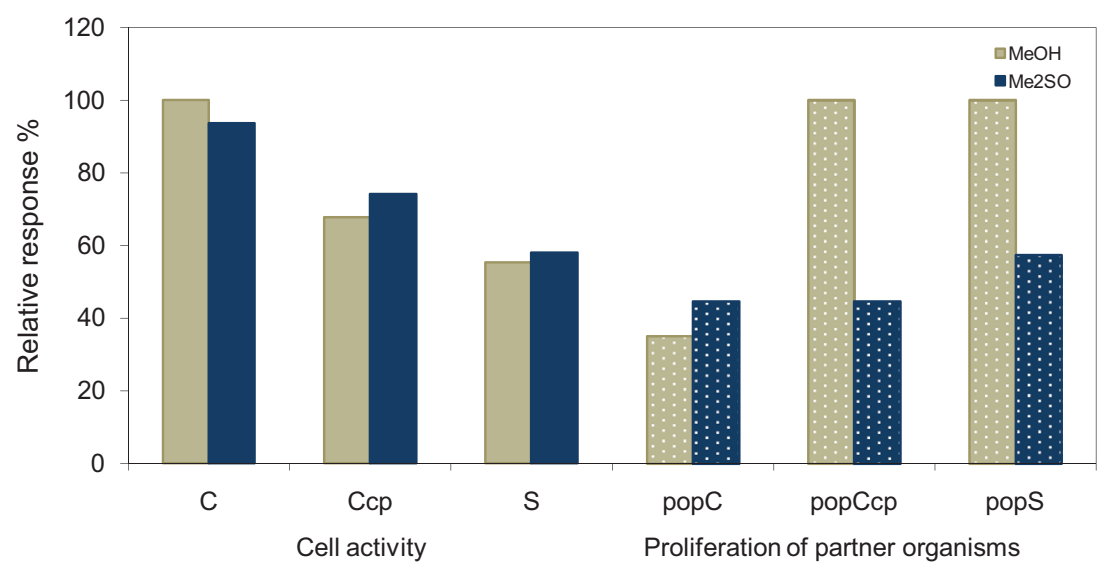

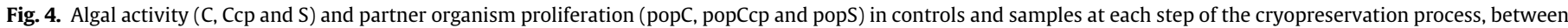

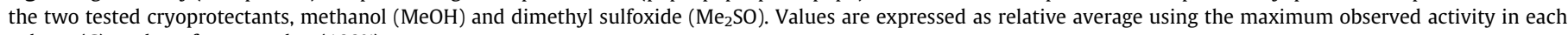
culture $(C)$ as the reference value (100\%).

model (with $m$ parameters) and ending with a simple univariate model presenting only one last parameter. In this iterative process, the least significant parameters (with the highest relative uncertainty) were successively removed, one at a time. Subsequently, based on the least squares approach, the parsimonious model was sequentially tested for parameter rejection and parsimony through an F-test. Fitting variance $\left(\sigma_{\text {fit }}^{2}\right)$ of a model with $\mathrm{m}$ parameters is given by:

$\sigma_{\text {fit }}^{2}(m)=\mathrm{SS}(m) / \mathrm{ndf}=\sum_{i=1}^{n}\left(y_{i}^{*}-y_{i}\right)^{2} /(n-m)$

where $y_{i}^{*}$ and $y_{\mathrm{i}}$ are the predicted and respective response value for each $n$ experimental data points. Comparing successively the simplified models, parsimony is obtained when a significant variance change occurs $(p<0.05)$, directly accessed via $p$-value calculation using 1 and $(n-m)$ degrees of freedom, respectively. The test value (TV) for model parsimony is obtained as:

$\mathrm{TV}=\Delta \mathrm{SS} /\left(\Delta \mathrm{ndf} \cdot \sigma_{\mathrm{pe}}^{2}\right)=[\mathrm{SS}(m-1)-\mathrm{SS}(m)] / \sigma_{\text {fit }}^{2}(m)$

where $\sigma_{\mathrm{pe}}^{2}=\sigma_{\mathrm{fit}}^{2}(m)$, is the pure error variance (Eq. (6)), SS $(m-1)$ and $\mathrm{SS}(m)$ stand for the sum of squares obtained in the simplified ( $m-1$ parameters) and previous ( $m$ parameters) models, which corresponds to a unitary variation in the number of degrees of freedom, $(\Delta \mathrm{ndf}=1)$.

In order to check the model ability to describe experimental response, the overall correlation coefficient was also evaluated for high correlation case $\left(H_{0}:|r|=1\right)$ using the $t$-test:

$\mathrm{TV}=|1-r| \cdot\left[(n-m) /\left(1-r^{2}\right)\right]^{1 / 2}$

\section{Results}

Regardless of the used cryoprotectant, a general trend for decreasing algal activity was observed along the cryopreservation process - both the addition of the cryoprotectant (Ccp) and the freezing/thawing $(\mathrm{S})$ are responsible for activity loss, leading to a final viability of about one half (Fig. 4).

\section{Statistical analysis by t-tests}

These observations were statistically tested through paired $t$-tests to detect significant differences between cryoprotectants at each cryopreservation step, and showed that there was signifi-
Table 2

Paired $t$-test values for algal activity and partner organism proliferation (pop) throughout the cryopreservation process, using $\mathrm{MeOH}$ and $\mathrm{Me}_{2} \mathrm{SO}$ as cryoprotectants. Highlighted in bold are $p$-values $<0.050$, corresponding to detected significant differences.

\begin{tabular}{llll}
\hline Responses & Tested comparisons & \multicolumn{2}{l}{-Value } \\
\cline { 3 - 4 } & & $\mathrm{MeOH}$ & $\mathrm{Me}_{2} \mathrm{SO}$ \\
\hline \multirow{2}{*}{ Cell activity } & Ccp-C & $\mathbf{0 . 0 1 0}$ & $\mathbf{0 . 0 2 2}$ \\
\multirow{2}{*}{ Pop } & S-Ccp & $\mathbf{0 . 0 3 2}$ & 0.325 \\
& popCcp-popC & $\mathbf{0 . 0 0 0}$ & - \\
& popS-popCcp & - & 0.169 \\
\hline
\end{tabular}

cant activity loss after cryoprotectant addition with both $\mathrm{MeOH}$ or $\mathrm{Me}_{2} \mathrm{SO}(p<0.05)$ (Table 2, $\left.\mathrm{Ccp}-\mathrm{C}\right)$. In the case of $\mathrm{MeOH}$, significant loss of activity occurred also after freezing/thawing $(p<0.05)$, as opposed to the case when $\mathrm{Me}_{2} \mathrm{SO}$ was applied. In this case, a slightly lower activity was observed in the projected data (Fig. 4) but this tendency is not statistically significant (Table 2, S-Ccp).

Pop increases drastically throughout the cryopreservation steps when $\mathrm{MeOH}$ is added to the cell suspension (popCcp), almost trebling after applying this cryoprotectant, but it remains unchanged after freezing (Fig. 4, popS). These observations are statistically supported $(p<0.05)$ (Table 2, popCcp-popC). However, when $\mathrm{Me}_{2-}$ SO is the used cryoprotectant, the pop (popCcp) remains unchanged (Fig. 4) (Table 2, popCcp-popC), and slight proliferation is observed after the freezing/thawing step (Fig. 4, popS), however with no statistical support given by the $t$-tests performed (Table 2 , popS-popCcp).

Regarding algal survival during the cryopreservation process, final cell viability (after freezing/thawing) is around half using either $\mathrm{MeOH}\left(55.4 \%\right.$ ) or $\mathrm{Me}_{2} \mathrm{SO}$ (62.1\%) (Fig. 5). Despite cell loss due to the cryoprotection step was higher with the addition of $\mathrm{MeOH}(32.3 \%)$ than with $\mathrm{Me}_{2} \mathrm{SO}$ (20.7\%), this adverse effect of $\mathrm{MeOH}$ appeared to be compensated during the freezing/thawing step $(12.4 \%$ and $17.2 \%$ cell loss respectively).

As previously stated, both tested cryoprotectants led to nearly half the number of viable cells at the end of the cryopreservation procedure. Since they were tested in statistically equivalent conditions concerning the variables (cell activity and pop), direct comparisons were provided by a $t$-test to estimate probabilities for $\mathrm{MeOH}-\mathrm{Me}_{2} \mathrm{SO}$ differences in respect to cell activity (C, Ccp, S) and pop (popC, popCcp, popS) at each treatment level. The result from the test confirmed that both cryoprotectants have similar impact over algal viability (previously seen in Fig. 4) and no other sig- 

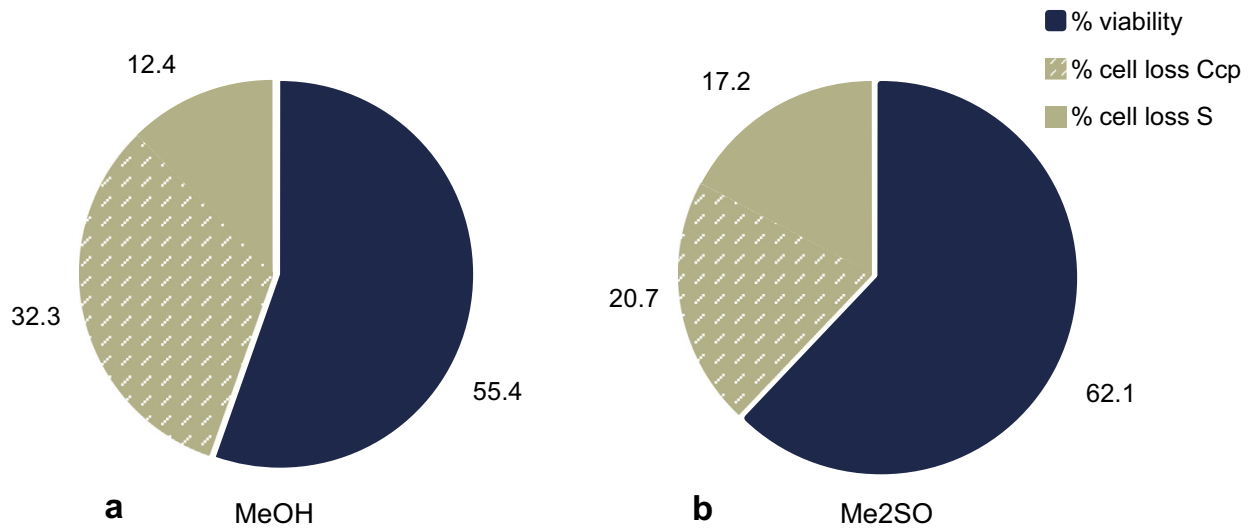

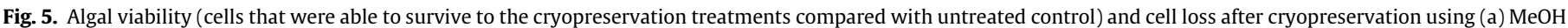
and (b) $\mathrm{Me}_{2} \mathrm{SO}$ as cryoprotectants. Values are expressed as percentages.

Table 3

Paired $t$-test values for algal activity $(\mathrm{C}, \mathrm{Ccp}, \mathrm{S})$ and partner organism proliferation (popC, popCcp, popS) between $\mathrm{MeOH}$ and $\mathrm{Me}_{2} \mathrm{SO}$.

\begin{tabular}{lll}
\hline Tested variable & $\mathrm{MeOH}-\mathrm{Me}_{2} \mathrm{SO}$ & $p$-Value \\
\hline Algal activity & $\mathrm{C}$ & 0.360 \\
& $\mathrm{Ccp}$ & 0.643 \\
& $\mathrm{~S}$ & 0.814 \\
& popC & 0.421 \\
Partner organism & popCcp & $\mathbf{0 . 0 0 6}^{\mathrm{a}}$ \\
Proliferation & popS & $\mathbf{0 . 0 0 4}^{\mathrm{a}}$ \\
\hline
\end{tabular}

a Significant differences $p<0.05$.

nificant differences were detected regarding algal activity at each cryopreservation step.

Nevertheless, high significant difference was found between the two used cryoprotectants regarding the pop, with the addition of $\mathrm{MeOH}$ having a stimulating effect to a level that remains almost unaffected by the freezing/thawing step $(p<0.05)$ (popCcp and pops, Table 3.

\section{Multivariate modeling}

Small, uni. and vac. are the cellular features tested for their relation to the experimental variables cell activity and pop (as explained in "Materials and methods"). Each experiment, with $\mathrm{MeOH}$ and $\mathrm{Me}_{2} \mathrm{SO}$, was separately tested and an optimized model retrieved information regarding the combination of the cryopreservation-inherent variables and the cell features. These parsimonious models were compiled (Table 4). In all cases, the overall correlation is very significant, reinforcing a good ability to describe the experimental data $\left(H_{0}:|r-1|=0\right)$.

Cell activity after cryoprotectant addition (Ccp) has the larger number of functional dependencies. Focusing on the study with $\mathrm{MeOH}$, it is seen that the most relevant factors affecting Ccp is popC ( -1.68$)$, followed by $C(1.12)$, while uni. and vac. have a much smaller impact, positive in the case of unicellular strains (0.26) and opposite when vacuoles were detected prior to cryopreservation $(-0.27)$ (Table 4). So, the initial population of partner organisms (popc) negatively affects the activity of cells after $\mathrm{MeOH}$ addition and greater activity of the initial culture (C) is related to its increase prior to cryopreservation. Regarding the final viability (S), it is mainly dependent on a single variable, $\mathrm{Ccp}$, with a positive dependence $(0.80)$ but lower than the unity, revealing a net loss of cell activity in the freezing/thawing step. Pop after cryoprotectant addition (popCcp) is positively affected by a higher presence initially in culture (popC), with a slight tendency for affecting unicellular algae (0.10). The level of pop detected after freezing/thawing (popS) remains unchanged since the cryoprotectant addition step (1.00 popCcp). When $\mathrm{Me}_{2} \mathrm{SO}$ was the used cryoprotectant, the most relevant coefficient for cell activity after its addition is the activity displayed prior to cryopreservation, i.e. the culture's initial density (C). A slight positive dependence was also shown for unicellular algae. Activity after freezing (S) depends exclusively on the activity shown after $\mathrm{Me}_{2} \mathrm{SO}$ addition (0.70). No alterations were found in the pop either after cryoprotectant addition or after the freezing/thawing step. The overall description of the cryopreservation-inherent variables and the cell features retrieved by the global model, shows that the activity after cryoprotection (Ccp), regardless of the used cryoprotectant, is positively related with the initial culture activity $(0.93 \mathrm{C})$, slightly promoted for unicellular cells ( 0.21 uni.). Cell activity after cryoprotection (Ccp) is negatively influenced by the presence of partner organisms in the initial culture $(-0.72 \mathrm{popC})$ and by the presence of vacuoles $(-0.16 \mathrm{vac}$.), although only a slight dependence was found in the latter. The model shows evident negative influence on the activity after cryoprotectant addition and $\mathrm{MeOH}$ is the cryoprotectant that contributes to this effect, as showed in the global model $(-0.19$ $\mathrm{MeOH})$. Cell activity after freezing is positively affected by the activity achieved after cryoprotectant addition (0.48 Ccp), especially for small sized cells. Finally, the proliferation of partner organisms after cryoprotection and also after freezing depends on

Table 4

Parsimonious models that define variable dependency regarding the cryopreservation steps and the cell features with $\mathrm{MeOH}$ and $\mathrm{Me} 2 \mathrm{SO}$ as cryoprotectants.

\begin{tabular}{|c|c|c|c|}
\hline Step & $\mathrm{MeOH}$ & $\mathrm{Me}_{2} \mathrm{SO}$ & Global \\
\hline Ccp & $1.12 \mathrm{C}-1.68 \mathrm{popC}+0.26 \mathrm{Uni}-0.27 \mathrm{Vac}$ & $-0.33+1.10 \mathrm{C}+0.25 \mathrm{Uni}$ & $0.93 \mathrm{C}-0.72 \mathrm{popC}+0.21 \mathrm{Uni}-0.16 \mathrm{Vac}-0.19 \mathrm{MeOH}$ \\
\hline $\mathrm{S}$ & $0.80 \mathrm{Ccp}$ & $0.70 \mathrm{Ccp}$ & $0.48 \mathrm{Ccp}+0.21$ Small \\
\hline pop Cср & 2.42 popC +0.10 Uni & 1.00 popC & $1.06 \mathrm{popC}+0.36 \mathrm{MeOH}$ \\
\hline pop $S$ & 1.00 рорСср & 1.00 рорс & $1.06 \mathrm{popC}+0.29 \mathrm{MeOH}$ \\
\hline
\end{tabular}


the detected presence in the initial culture (popC) and the use of $\mathrm{MeOH}$ evidences it.

\section{Discussion}

The recovery of cryopreserved algae after 20 years of storage was evidenced years ago [8] and recent studies found that algae may be kept for 15 years with constant rates of viability and chlorophyll-a content [25]. No specific indications of the state of the culture regarding axenicity were found in either of these publications or in the wider literature. However, searching the available database of the reference collection from where the studied strains were obtained, it was found that the authors had evaluated at least one axenic and one non-axenic strain, which kept the same stable profile for viability and chlorophyll content during the study. This indicates that by successfully cryopreserving the biological consortia in culture (i.e. algal cells and their partner organisms) the stability of the algae is maintained.

Previously to this study, routine cryopreservation of ACOI microalgae frequently led to the observation of opacity on the algae recovered after cryopreservation with $\mathrm{MeOH}$ but not as evident when $\mathrm{Me}_{2} \mathrm{SO}$ was used. This observation indicates pop, but it was not previously considered as relevant, because it usually disappeared when the algal cells recovered a normally dense culture. The hypothesis rising from this observation was that the choice of the optimal cryoprotectant might have implications beyond preventing cryoinjury, such as a possible interference with the culture as a whole, including the partner organisms. To test this hypothesis, strains were selected within the ACOI collection from different taxa and origins, with a common feature of being mucilage producers. The choice of this criterion was based on the presumption that mucilage production would exacerbate the non-axenicity-related effect of pop during cryopreservation.

Our results showed that part of the cell loss during the cryopreservation process was due to the cryoprotectant addition (20.7-32.3\% loss). The use of a cryoprotectant has two sides: used in the optimized concentration/exposure time it will prevent cryoinjury due to solution effects and ice crystal formation; when used in non-optimized conditions it can also be toxic [14,5]. The optimal use of cryoprotectants lies in the combination of high cryoprotective ability with low toxicity [4], however this is theoretical and so far it is determined empirically because each cell type has its own specificities, which will be reflected in the cryoprotectant action [17]. In our work, both $\mathrm{MeOH}$ and $\mathrm{Me}_{2} \mathrm{SO}$ were efficient cryoprotectants, leading to the successful cryopreservation of about half the algae in culture, 55.4-62.1\%, but $\mathrm{MeOH}$ also promotes pop while in the $\mathrm{Me}_{2} \mathrm{SO}$ case this was not observed. Statistical tests showed that significant differences regarding pop are only evident when this effect was analyzed after the addition of the cryoprotectant. A severe increase in pop due to $\mathrm{MeOH}$ was shown and statistically supported $(p<0.05)$. Furthermore it was shown that this adverse effect of $\mathrm{MeOH}$ of triggering pop is later compensated after freezing/thawing step because it causes less cell loss comparing to $\mathrm{Me}_{2} \mathrm{SO}$.

Modeling allowed us to quantify the relative effect on algal activity of the cryoprotectants, pop, and the cell features. Since the statistical study was based on the average data collected from the tests on nine different mucilaginous strains, the results reflect the cryoprotectant effect on mucilaginous non-axenic algae in general, rather than for each strain in particular. The main conclusions from the models are as follows: (1) regardless of the cryoprotectant used, denser cultures of unicellular algae are more able to survive cryoprotectant toxicity, maybe due to the greater probability of resistant microalgae being present, especially if they are simple unicellular cells, in which the exchange between water and cryo- protectant is more efficient; (2) when MeOH is used as a cryoprotectant, cells with vacuoles at the time of cryopreservation show slightly lower ability to survive following the addition of cryoprotectant. Maybe this is due to the higher water content inside the cell, that potentially limits an efficient cryo-dehydration action as a consequence of extracellular freezing and colligatively acting $\mathrm{MeOH}$ entering the cell and substituting the water molecules; (3) when $\mathrm{Me}_{2} \mathrm{SO}$ is used there is no clear evidence of the negative impact of popC and vac. over Ccp, but a negative constant is present in the parsimonious model that may be interpreted as a statistical effect of an undetermined influence of these factors; (4) the final cell viability is independent of the used cryoprotectant, it is higher when more cells survive cryoprotection, as expected, and there is a trend for higher viability when the cryopreserved cells are of the small size type and with no vacuoles at the time of cryopreservation. This trend has been repeatedly observed in the past for the cryopreservation of microalgal cultures, especially from culture collections (e.g. [27]) but no statistical model has been previously applied. We can interpret this by the two-factor hypothesis used for explaining cryoinjury [22]: theoretically, small cells with low water content (e.g. with no vacuoles) are more able to undergo the necessary exchange of water leaving the cell and $\mathrm{MeOH}$ entering it [33], in order to create an ice crystal-safe environment as a consequence of extracellular freezing establishing a water vapour deficit between the inside and outside of the cell. In this way they cryo-dehydrate more efficiently while freezing, where the cryoprotection step is more successful and the cells survive better the physical-chemical freezing injuries.

Regarding the partner organisms, the detectable level in the initial culture remains almost unchanged after the addition of cryoprotectant unless $\mathrm{MeOH}$ is used. When this cryoprotectant is used, pop is also slightly related to the unicellular-type of cryopreserved algae, possibly due to the fact that an individual mucilaginous sheath may be more prone to develop fungal mycelia and/ or bacteria than a colony-type, if we consider the relative area of exposure.

One aspect that favors pop after freezing is the possibility that the cell content released by disrupted algal cells during cryopreservation may be used by partners, thus broadening the available nutrients for their proliferation [15]. Since cryopreserved cells recover slower than controls (non-cryopreserved cells), the few that do survive may not be able to compete with high numbers of proliferating partner organisms [9].

Finally, we found that pop levels in cultures that went through the whole process of cryopreservation are dependent on the cryoprotectant used. When $\mathrm{Me}_{2} \mathrm{SO}$ is used, the proliferation depends exclusively on the detectable presence of the partners, while with $\mathrm{MeOH}$ it depends upon the existing level of pop after the addition of cryoprotectant. By using the information of the global model, we found evidence that the final pop is dependent on the initial pop and the addition of $\mathrm{MeOH}$, which reinforces the idea that $\mathrm{MeOH}$ has a dramatic effect on the proliferation, regardless of the level of partner organisms initially present. One possible explanation for this effect of $\mathrm{MeOH}$ is that the partners may preferentially use it as a source of energy and carbon. Another possibility is that the algal cells may be differentially stressed by $\mathrm{MeOH}$ but not the partners. A more complex explanation is that $\mathrm{MeOH}$ makes the dissolved nutrients more available to partner organisms for example by solubilizing them or by increasing their permeability.

Regarding the negative impacts of non-axenicity, cryopreservation programs for the conservation of specific algae, for example type material or other important algae, suggest that axenicity is mandatory when AFLP genomic consistency tests are included [10], however this aspect is usually not assertively considered as crucial in the majority of the published work on cryopreservation. For PCR-based taxonomic and phylogenetic work on microalgal 
cultures, non-axenicity is generally not considered as a problem. Molecular study of $18 \mathrm{~S}$ rRNA and $r b c L$ genes from DNA extracted from non-axenic ACOI eustigmatophytes cultured in liquid medium is ongoing [2], all BLAST searches retrieved similarities with eustigmatophytes. This is a concrete indication that bacterial or fungal DNA does not interfere with PCR, as long as the used primers are specific for microalgae. The bacterial 16S rRNA gene is not amplified with primers designed for 18S rRNA gene and the plastid $r b c L$ gene is absent in bacteria and fungi. The fungal 18S rRNA gene could be amplified but the use of specific primers for our target taxa prevented this event. Frequently, when the presence of bacterial DNA hinders the detection of the organism under study, specific primers are designed [26]. For barcoding projects nuclear spacer genes such as ITS2 are used, so the presence of DNA from partners may be somehow problematic [31]. Also in this case, specific primers can be used or ultimately the culture may be axenicized for that purpose. For genome sequencing projects, alternative approaches to achieve symbiont-free sequence was obtained for macroalgae [24] and new strategies must be designed for microalgae too. When industrial purposes are intended for the large-scale cultivation of microalgae, a new trend towards the idea of cultivating the consortia is emerging from studies such as the symbiotic association of bacteria and fungi in the widely cultivated microalgal genus Chlorella Beijerinck [34].

\section{Concluding remarks}

The cryoprotection step triggers the proliferation process when $\mathrm{MeOH}$ is used and the level of pop remains unaltered after freezing/thawing. $\mathrm{Me}_{2} \mathrm{SO}$ has no statistically significant effect on pop for non-axenic microalgae, cultured under the tested conditions. Further studies are needed to understand the mechanisms underlying this effect; we consider that the most plausible explanation is that $\mathrm{MeOH}$ is used by partner organisms as nutrient source in culture.

In summary, the axenicity status of the cultures to be cryopreserved is not crucial for the success of the procedure. Nevertheless, it should be taken into account when decisions are made to cryopreserve sets of non-axenic algae from different taxa in a routine basis: $\mathrm{Me}_{2} \mathrm{SO}$ is the correct choice to prevent pop, especially when the algae have a mucilaginous sheath.

Although there is no consensus about how to deal with the coisolated organisms in culture collections, our position is that they must be kept intact. However, it is advisable to always report the axenic/non-axenic state, as a default rule. Partner organisms and the microalgae present in culture are considered as a consortium and strategies to cope with this must be integrated in cryopreservation methods. Another relevant aspect is the risk of spreading contamination from pop while cryostoring non-axenic cultures. Management of such cryobanks should evolve towards the integration of the known aspects related to cryopreservation-mediated contamination [5] in risk control protocols for long-term storing, thawing and the provision of biomaterials by the service sector.

The supply services of cultured microalgae for studies that require axenic strains must be accompanied with information of the axenicity status. There are two options: (i) the removal of partners is considered as a preliminary task to be performed by the purchaser prior to the study or (ii) it may be included as a specific service requirement for the provider collection prior to forwarding the culture. The compulsive removal of the co-isolates in all strains is not advisable, and sub-culturing of non-axenic cultures must always be performed in the flow cabinet to guarantee that no exogenous contaminant organisms join the initially isolated consortium. Therefore, for specific projects requiring axenic cultures, the culture collection service may engage in cleaning small groups of strains, always keeping the original non-axenic strain as the collection isolate. Special care must be taken with mucilaginous algae, because partners are attached to the sugar coat, usually in a symbiotic fashion [18]. The standardization of quality methods that include the information management of the axenicity status of cultures, are being developed to enable interoperability between different culture collections and laboratories using cultured microalgae [6]. The development of this good practice reinforces a growing trend of dealing with this aspect from a realistic point of view.

Insights on the symbiotic interactions are currently being revealed that are beginning to have considerable impact on the methods used for cultivation of microalgae [34]. However, more studies are required to clarify the significance of the interaction between all organisms in culture, how it may be affected by cryopreservation and how it interferes with the cryopreserved algae in the long term. Another aspect that requires further examination is the possibility that the population may shift following post thawing of the culture to a more complex composition than the homogeneous proliferation of all partners, with consequences for microalgal recovery. A metagenomic analysis may well shed some light on the diversity of co-isolates during pre and post-cryopreservation stages.

\section{Acknowledgments}

The authors acknowledge financial support provided by the Portuguese National Science and Technology Foundation (FCT) for the research project PTDC/BIA-QOR/71319/2006 and for the Ph.D. grant SFRH/BD/73359/2010 to Raquel. Special thanks are due to Dr. Erica Benson and Dr. Keith Harding for encouraging this research and for reviewing the manuscript and to Dr. Marek Eliáš for a fruitful discussion of axenicity impact on molecular work. Comments and suggestions from two referees greatly contributed to improve this manuscript.

\section{References}

[1] R. Amaral, M.F. Santos, L.M.A Santos, Overcoming recalcitrance in Porphyridium aerugineum Geitler employing encapsulation-dehydration cryopreservation methods, Cryo Lett. 30 (6) (2009) 462-472.

[2] R. Amaral, M. Eliáš, L.M.A. Santos and M.F. Santos, Molecular and microscopic evidence place microalgal species of Characiopsis into the Eustigmatophyceae, Poster, 5th European Phycological Congress, Rhodes, Greece, Eur. J. Phycol. 46 (Suppl.1) (2011) 109-194.

[3] S.A. Amin, D.H. Green, M.C. Hart, F.C. Küpper, W.G. Sunda, CJ. Carrano, Photolysis of iron-siderophore chelates promotes bacterial-algal mutualism, PNAS 106 (2009) 17071-17076.

[4] A. Baudot, V. Odagescu, Thermal properties of ethylene glycol aqueous solutions, Cryobiology 48 (2004) 283-294.

[5] E.E. Benson, Cryopreservation of phytodiversity: a critical appraisal of theory \& practice, Crit. Rev. Plant Sci. 27 (2008) 141-219.

[6] E.E. Benson, F. Betsou, R. Amaral, L.M.A. Santos, K. Harding Standard PREanalytical codes (SPREC): a new paradigm for environmental biobanking sectors explored in algal culture collections, Biopreserv. Biobanking 9 (4) (2011) 399-410.

[7] M.T. Croft, A.D. Lawrence, E. Raux-Deery, M.J. Warren, A.G. Smith, Algae acquire vitamin $B_{12}$ through a symbiotic relationship with bacteria, Nature 438 (2005) 90-93.

[8] J.G. Day, M.M. Watanabe, G.J. Morris, R.A. Fleck, M.R. McLellan, Long-term viability of preserved eukaryotic algae, J. Appl. Phycol. 9 (1997) 121-127.

[9] J.G. Day, K. Harding, Cryopreservation of algae, in: B.M. Reed (Ed.), Plant Cryopreservation - A Practical Guide, Springer, Netherlands, 2007, pp. 95-116.

[10] J.G. Day, T. Pröschold, T. Friedl, M. Lorenz, P. Silva, Conservation of microalgal type material: approaches needed for 21st century science, Taxon 59 (1) (2010) 3-6.

[11] M.R. Droop, A procedure for routine purification of algal cultures with antibiotics, Br. Phycol. Bull. 3 (1967) 295-297.

[12] M.R. Droop, K.G.R. Elson, Are pelagic diatoms free from bacteria?, Nature 211 (1966) 1096-1097

[13] D.J. Dumet, E.E. Benson, The use of physical and biochemical studies to elucidate and reduce cryopreservation-induced damage in hydrated/ desiccated plant germplasm, in: F. Engelmann, H. Takagi (Eds.), 
Cryopreservation of Tropical Plant Germplasm: Current Research Progress and Application, International Plant Genetic Resources Institute, Rome, pp. 43-56.

[14] K. Harding, J.G. Day, M. Lorenz, H. Timmermann, T. Friedl, D.H. Bremner, E.E. Benson, Introducing the concept and application of vitrification for the cryoconservation of algae - a mini-review, Nova Hedwigia 79 (2004) 207-226.

[15] K. Harding, J. Müller, H. Timmermann, M. Lorenz, J.G. Day, T. Friedl, Encapsulation dehydration colligative cryoprotective strategies and amplified fragment length polymorphism markers to verify the identity and genetic stability of euglenoids following cryopreservation, Cryo Lett. 31 (6) (2010) 460-472.

[16] K. Hirata, M. Phunchindawan, J. Tukamoto, S. Goda, K. Miyamoto, Cryopreservation of microalgae using encapsulation-dehydration, Cryo Lett. 17 (1996) 321-328.

[17] Z. Hubálek, Protectants used in the cryopreservation of microorganisms, Cryobiology 46 (2003) 205-229.

[18] A.K. Jones, M.E. Rhodes, S. Evans, The use of antibiotics to obtain axenic cultures of algae, Br. Phycol. J. 8 (1973) 185-196.

[19] B.S.C. Leadbeater, Tribute: the 'droop equation' - Michael Droop and the legacy of the 'cell-quota model' of phytoplankton growth, Protist 157 (2006) 345-358.

[20] E. Lešková, J. Kubíková, E. Kováiková, M. Košická, J. Porubská, K. Holíková, Vitamin losses: retention during heat treatment and continual changes expressed by mathematical models, J. Food Compos. Anal. 19 (2006) 252-276.

[21] L.M. Márquez, R.S. Redman, R.J. Rodriguez, M.J. Roossinck, A virus in a fungus in a plant: three-way symbiosis required for thermal tolerance, Science 315 (2007) 513-515

[22] P. Mazur, S.P. Leibo, E.H.Y. Chu, A two-factor hypothesis of freezing injury evidence from Chinese hamster tissue-culture cells, Exp. Cell Res. 71 (1972) $345-355$.

[23] G.J. Morris, The cryopreservation of Chlorella. 1. Interactions of rate of cooling, protective additive and warming rate, Arch. Microbiol. 107 (1976) 57-62.

[24] Y. Nakamura, N. Sasaki, M. Kobayashi, N. Ojima, M. Yasuike, Y. Shigenobu, M. Satomi, Y. Fukuma, K. Shiwaku, A. Tsujimoto, T. Kobayashi, I. Nakayama, F. Ito,
K. Nakajima, M. Sano, T. Wada, S. Kuhara, K. Inouye, T. Gojobori, K. Ikeo, The first symbiont-free genome sequence of marine red alga, susabi-nori (Pyropia yezoensis), PLoS ONE 8 (3) (2013) e57122.

[25] K. Nakanishi, K. Deuchi, K. Kuwano, Cryopreservation of four valuable strains of microalgae, including viability and characteristics during 15 years of cryostorage, J. Appl. Phycol. 24 (2012) 1381-1385.

[26] A. Namba, Y. Shigenobu, M. Kobayashi, T. Kobayashi, I. Oohara, A new prime for 16S rDNA analysis of microbial communities associated with Porphyra yezoensis, Fish. Sci. 76 (2010) 873-878.

[27] H.C. Osório, C.N. Laranjeiro, L.M.A. Santos, M.F. Santos, First attempts to cryopreserve strains from the Coimbra Collection of Algae (ACOI) and the use of image analysis to assess viability, Nova Hedwigia 79 (2004) 227-235.

[28] T.P. Ryan, Modern Regression Methods, second ed., Wiley, New Jersey, 2009.

[29] L.M.A. Santos, M.F. Santos, The coimbra culture collection of algae (ACOI), Nova Hedwigia 79 (2004) 39-47.

[30] U.G. Schlösser, SAG-sammlung von algenkulturen at the University of Göttingen Catalogue of strains, Bot. Acta 107 (1994) 113-186.

[31] R.F. Stern, R.A. Andersen, I. Jameson, F.C. Küpper, M.-A. Coffroth, D. Vaulot, F. Le Gall, B. Véron, J.J. Brand, H. Skelton, F. Kasai, E.L. Lilly, P.J. Keeling, Evaluating the ribosomal internal transcribed spacer (ITS) as a candidate dinoflagellate barcode marker, PLoS ONE 7 (8) (2012) e42780.

[32] H. Ueda, S. Otsuka, K. Senoo, Community composition of bacteria co-cultivated with microalgae in non-axenic algal cultures, Microbiol. Cult. Coll. 25 (1) (2009) 21-25.

[33] J.R. Walsh, K.R. Diller, J.J. Brand, Measurement and simulation of water and methanol transport in algal cells, J. Biomech. Eng. 126 (2) (2004) 167-179.

[34] K. Watanabe, N. Takihana, H. Aoyagi, S. Hanada, Y. Watanabe, N. Ohmura, H. Saiki, H. Tanaka, Symbiotic association in Chlorella culture, FEMS Microbiol. Ecol. 51 (2005) 187-196. 